\title{
Glycemic Control and its Predictors among Adult Diabetic Patients attending Mettu Karl Referral Hospital, Southwest Ethiopia: A Prospective Observational Study
}

Tadesse Sheleme (D) - Girma Mamo • Tsegaye Melaku • Tamiru Sahilu

Received: May 16, 2020 / Published online: June 24, 2020 (C) The Author(s) 2020

\section{ABSTRACT}

Introduction: The current estimate is that 463 million people worldwide have diabetes. In 2017, an estimated 5 million (9.9\%) deaths worldwide among adults were caused by diabetes. The burden of disease associated with uncontrolled diabetes is substantial in terms of mortality and cardiovascular disease. The aim of this study was to assess glycemic control level and its predictors among adult patients with diabetes.

Methods: A prospective observational study was conducted among patients with diabetes during follow-up at an ambulatory clinic of Mettu Karl referral hospital from 15 April to 09 August 2019. The consecutive sampling method was used to collect data, following which the data were entered into Epidata manager version

Digital Features To view digital features for this article go to https://doi.org/10.6084/m9.figshare.12459182.

T. Sheleme $(\bowtie)$

Department of Pharmacy, College of Public Health and Medical Science, Mettu University, Mettu, Ethiopia

e-mail: tadeshe14@gmail.com

G. Mamo · T. Melaku

School of Pharmacy, Institute of Health, Jimma

University, Jimma, Ethiopia

T. Sahilu

Department of Pharmacy, College of Health Science, Assosa University, Assosa, Ethiopia
4.4.2 and exported to the SPSS version 24.0 statistical software package for analysis. Logistic regression analysis was performed to identify predictors of poor glycemic control. Variables whose significance level was $<0.05$ ( $p$ value) were considered to be predictors of poor glycemic control.

Results: A total of 330 diabetic patients were included in the study, among whom 240 $(72.7 \%)$ had poor glycemic control. The predictors of poor glycemic control in the multivariate logistic regression analysis were overweight [adjusted odds ratio (AOR) 4.07; $95 \%$ confidence interval (CI) 1.60, 10.36; $p=0.003]$, obesity (AOR 4.39; 95\% CI 1.59, $12.14 ; p=0.004)$, higher estimated glomerular filtration rate (eGFR) (AOR 2.34; 95\% CI 1.23, $4.44 ; p=0.010$ ), type 1 diabetes (AOR 3.22; 95\% CI $1.58,6.55 ; p=0.001)$, poor diet adherence (AOR 6.95; 95\% CI 3.63, 13.32; $p<0.001$ ) and non-adherence to medications (AOR 5.82; 95\% CI 2.77, 12.26; $p<0.001$ ).

Conclusion: Almost three-quarters of the study population of diabetic patients had poorly controlled blood sugar. Overweight, obesity, higher eGFR, type 1 diabetics, poor adherence to diet recommendation and non-adherence to medications were independent predictors of poor glycemic control. Educational strategies should focus on improving adherence to the recommended diet and medication(s), achieving weight control and optimizing glycemic control. 
Keywords: Diabetes; Ethiopia; Glycemic control; Predictors; Mettu Karl Referral Hospital

\section{Key Summary Points}

Why carry out this study?

The prevalence of diabetes, which is associated with a higher risk for serious life-threatening health conditions, is rapidly increasing worldwide.

Uncontrolled diabetes is associated with a substantial mortality and morbidity, including cardiovascular disease burden.

The aim of this study was to assess glycemic control level and its predictors among patients with diabetes.

\section{What was learned from the study?}

Almost three-quarters of the study population of diabetic patients had poorly controlled blood sugar.

Independent predictors of poor glycemic control were overweight, obesity, higher estimated glomerular filtration rate, type 1 diabetics, poor adherence to recommended diet and non-adherence to medications.

Educational strategies should focus on enhancing adherence to recommended diet and medications and achieving weight control in order to optimize glycemic control in this population.

\section{INTRODUCTION}

Diabetes mellitus, a chronic metabolic disorder caused by defects in insulin secretion and/or action, is characterized by hyperglycemia. Its prevalence worldwide is rapidly increasing [1], as reported by the World Health Organization which estimated that, globally, 422 million adults aged $>18$ years were living with diabetes in 2014, compared to 108 million in 1980 [2]. In
2019, The International Diabetes Federation (IDF) estimated that there are 463 million people with diabetes worldwide, representing 9.3\% of the global adult population (age 20-79 years). This number is expected to increase to 578 million (10.2\%) in 2030 and to 700 million $(10.9 \%)$ in 2045 [3]. More specifically, the prevalence of diabetes is increasing in developing countries due to population growth, aging populations, unhealthy diets, obesity and sedentary lifestyles [4]. The obstacles to addressing diabetes in developing countries, particularly those in Sub-Saharan Africa, are a limited understanding of its prevalence, modifiable risk factors and complications arising from under-recognition and/or under-treatment [5]. Ethiopia has been found to have the highest number of people with diabetes in Africa, with an estimated 2.6 million people with diabetes [6].

People with diabetes have an increased risk of developing a number of serious life-threatening health conditions, resulting in higher medical care costs, reduced quality of life and increased mortality [7]. The global healthcare expenditure on people with diabetes was estimated to be 850 billion U.S. dollars (USD) in 2017. Diabetes contributed directly to approximately 5 million (9.9\%) deaths worldwide among people aged 20-99 years in 2017, with about $36.5 \%$ or 1.8 million of these deaths occurring in people aged $<60$ years. It also was associated with $6.0 \%$ of all-cause mortality in the African region [8]. One study revealed that $5.0 \%$ of deaths in Ethiopia were attributable to diabetes [9].

Globally, about $40.0-60.0 \%$ of patients with diabetes have poorly controlled blood glucose [10]. One study conducted in Sub-Saharan Africa showed that $74.0 \%$ of patients with diabetes had poor glycemic control [11] and that in Ethiopia specifically, a large proportion of patients with diabetes (65.6-66.8\%) were not able to achieve good glycemic control [6]. Glycemic control remains a challenging task, with the majority of patients failing to achieve a good glycemic status. It is affected by factors such as age, gender, level of education, body mass index (BMI), alcohol, dietary habits, home blood glucose monitoring, physical exercise, 
duration of diabetes, family history of diabetes, medications taken and numerous other factors $[10,12]$.

Uncontrolled diabetes is associated with a substantial mortality and cardiovascular disease burden [13]. It has been reported that uncontrolled diabetes has a greater impact on morbidity and mortality related to the disease in Sub-Saharan Africa than in any other region in the world [14]. In Ethiopia, complications arising from diabetes are higher among those patients with poor glycemic control [15]. Long duration of diabetes and poor glycemic control increase the chances of diabetes-related complications [16], and uncontrolled diabetes can lead to a number of acute and chronic complications as well as worsen the quality of life $[17,18]$.

Many studies have been conducted to explore the determinants of glycemic control, with the results showing that glycemic control is affected by factors such as longer duration of diabetes, low level of education, lack of information on diabetes, lower income, poor compliance to diet and medication(s), poor attitude towards the disease, poor self-management behavior, sedentary living, overweight/obesity, renal impairment, hypertension and dyslipidemia $[10,19,20]$. It has also been reported that poverty and lack of knowledge affect the control of diabetes in rural communities [21]. The association between overweight or obesity, gender and age of patients, respectively, and glycemic control has been the aim of a number of studies, with conflicting results. For example, one study from the USA showed that obesity and overweight were strongly associated with poor glycemic control [22], while a study from Asia found no relationship between glycemic control and BMI, waist circumference or central obesity [23]. It has also been reported that being female was associated with poor glycemic control [24, 25], but Mahmoud et al. [26] found that being male was associated with poor glycemic control. Some studies demonstrated that glycemic control was more difficult to obtain with increasing age $[25,27,28]$, a result that is inconsistent with results from other studies which indicated that younger age was associated with poor glycemic control [24, 26, 29].
The glycemic level of the majority of diabetic patients in Ethiopia has been reported to be poorly controlled [30-33]. One study conducted at the Jimma University Medical Center found that $59.4 \%$ patients had poor glycemic control [30]. Another study performed in Addis Ababa showed that $80 \%$ of patients had poor glycemic control [31], while studies conducted in Dessie and Gondar reported that $70.8 \%$ and $64.7 \%$ of patients with diabetes had poor glycemic control, respectively $[32,33]$. Others studies in Ethiopia have focused on the factors associated with poor glycemic control. These studies found that age, income, triglyceride and low-density lipoprotein levels and glucometer non-use were significantly associated with poor glycemic control [34] and that factors such as low educational level, longer duration of diabetes, being a farmer or merchant, combination of insulin and oral medication and poor medication adherence, were associated with poor glycemic control $[32,35]$.

The aim of diabetes management is to prevent mortality and complications by optimizing the blood glucose level [36]. Despite the advanced scientific knowledge in diagnostic and treatment modalities, a high proportion of patients with diabetes still have poorly controlled blood glucose [20, 37]. The reasons underlying this failure to achieve optimal glycemic control are complex and multifactorial [38]. Consequently, early identification of people living with diabetes who are at risk for the complications of this disease is important as early disease management with the appropriate intervention can help to prevent or delay the burden associated with these complications.

Although a number of studies conducted in Ethiopia have reported a high prevalence of poor glycemic control among patients with diabetics, more studies are needed to generalize glycemic control level and its predictors at the national level. To our knowledge, no study has been conducted to date at the Mettu Karl referral hospital with the aim to determine glycemic control and its predictors. All previous studies performed to evaluate glycemic control and its determinants in Ethiopia were crosssectional studies [30-35]. The study reported here is a prospective observational study with 
the aim to determine glycemic control levels among patients with diabetes in Ethiopia and its predictors.

\section{METHODS}

\section{Study Setting and Period}

The study was conducted from 15 April to 09 August 2019 at the ambulatory clinic of Mettu Karl referral hospital, Oromia region, southwest Ethiopia. The hospital is located in the town of Mettu, located $600 \mathrm{~km}$ southwest of Addis Ababa, the capital city of Ethiopia. It serves about 2.5 million people from Ilu Ababor Zone, Gambella Regional State and the adjacent Southern Nation and Nationalities Peoples Region. The healthcare service provided by the hospital includes an outpatient department, inpatient services, a critical care unit (intensive care unit) and an emergency intervention unit. The hospital also has medical, surgical and gynecological referral clinics. Overall, the hospital provides healthcare services to approximately 13,453 inpatients and 80,000 outpatients per year.

\section{Study Design and Population}

This study was a healthcare facility-based prospective observational study. The source population was all patients attending follow-up at an ambulatory clinic of Mettu Karl referral hospital. The study population comprised all adult patients with diabetes who attended the ambulatory clinic of Mettu Karl referral hospital during the data collection period and fulfilled the inclusion criteria.

\section{Eligibility Criteria}

Patients with type 1 and type 2 diabetes aged $\geq$ 18 years who had started taking antidiabetes medications were included in the study. The 1-month follow-up schedule for data collection was used to avoid including the same cases/patients repeatedly in the analysis as the majority of patients revisit the clinic every 1 month. The exclusion criteria were patients with diabetes unwilling to participate in the study and patients who were not fasting.

\section{Sample Size Determination and Sampling Technique}

The proportion of patients with diabetes with poor glycemic control was $59.4 \%$, as reported from an earlier study conducted at the Jimma university medical center among the diabetes patient population [30]. The required sample size was estimated using the single-population formula:

$$
n=\frac{(Z \alpha / 2)^{2} P(1-p)}{d^{2}}
$$

where $n=$ the desirable sample size, $Z_{\alpha / 2}=1.96$ (the critical value at $95 \%$ level of significance), $p=0.594$ (proportion of patients with poor glycemic control), $d=0.05$ (level of precision/ acceptable marginal error) and $q=1-p$. Replacing containers with values results in:

$$
n=\frac{(1.96) 2(0.594)(0.406)}{(0.05) 2}=371
$$

The total number of patients with diabetes attending follow-up visits at Mettu Karl referral hospital was 1560 . Therefore, the number of the source population $(N)$ was 1560 . Since the target population was below 10,000 , a finite correction formula could be applied to obtain a working or final sample size $(n f)$ :

$$
n f=\frac{n}{1+\frac{n}{N}}=\frac{371}{1+\frac{371}{1560}}=300
$$

An extra $10 \%$ was added to this number to allow for non-response; thus, the final sample size was $330(300+30)$.

All patients with type 1 and 2 diabetes attending follow-up visits at the ambulatory clinic of Mettu Karl referral hospital during the visiting hours of the clinic and who met the eligible criteria were enrolled in the study. The consecutive sampling method was used to collect data from patients with diabetes who met the inclusion criteria. 


\section{Study Variables}

The dependent variable was glycemic control status. The independent variables included socio-demographic variables (age, sex, educational status, residence, income, occupation and BMI), clinical factors (duration of diabetes, family history of diabetes, type of diabetes, comorbidities and estimated glomerular filtration rate [eGFR]), medication-related variables (type of antidiabetes medication and polypharmacy) and diabetes self-care practice and knowledge factors (self-monitoring of blood glucose [SMBG], adherence to antidiabetes medication, knowledge of target blood sugar, personal knowledge of diabetes, diet adherence, physical activity, attendance at diabetic educational session at health facility, alcohol use, chewing/ not chewing chat [also known as khat or qat] and smoking status).

\section{Validating Methods of Outcome}

The treatment outcome of the diabetes patients participating in the study was assessed in terms of glycemic control, assessed using fasting blood sugar (FBS) level. Prior to the collection of a blood sample for the laboratory test, the patient was asked to guarantee that he/she had fasted for a minimum of $8 \mathrm{~h}$ immediately prior to the visit. At each visit the patient was reminded once again of the requirement to fast for a minimum of $8 \mathrm{~h}$ prior to the laboratory test to be conducted at the following visit to the outpatient clinic. The baseline FBS was obtained on the first day that the patient visited the hospital during the study period. Month- 1 , month- 2 and month-3 FBS measurements were made at visits during the first, second and third month following the initial visit during the study period, respectively; and the average of these FBS measurements for the three consecutive months was used in the analysis. The glycemic status was categorized as good glycemic control if the average FBS was $80-130 \mathrm{mg} / \mathrm{dl}$ and poor glycemic control when $>130 \mathrm{mg} / \mathrm{dl}$. This categorization of glycemic control status is based on the recommendation in the American Diabetes Association guideline [39].

\section{Data Collection Instrument}

A structured questionnaire and the abstraction format were used to collect data. This method was developed following a review of the relevant literature [30-35]. The contents of the questionnaire and data extraction format include patient details, clinical investigations, medications, comorbidities, complications, selfcare practice, duration of diabetes mellitus and other disease-related issues. The data abstraction format was used to check and collect information on medications the patient was currently taking, laboratory values and diseaserelated data. Self-care activities, such as diet, physical activity, SMBG and adherence to antidiabetes medications, were assessed using the Summary of Diabetes Self-Care Activities [40]. Patient's knowledge of diabetes was measured using the 24-item version of the Diabetes Knowledge Questionnaire [41]. The data were collected by healthcare professionals (two nurses and two laboratory technologists) and supervised by two pharmacists.

\section{Data Collection Process}

The questionnaire was translated from English to local languages, such as Afan Oromo and Amharic. Patient data, such as socio-demographic characteristics, medication adherence, self-care activities, knowledge of diabetes and family history of diabetes, were collected in an interview with each patient. Patients were also asked about smoking status, alcohol consumption and whether or not they chewed chat. Clinical data, current therapeutic regimen and some laboratory values were obtained from each patient's medical records.

\section{Laboratory Measurements}

Laboratory tests were performed by laboratory technologists. Blood sampling consisted of drawing $5 \mathrm{ml}$ of blood from the antecubital vein under aseptic conditions using a sterile disposable syringe, collecting the blood sample into clean and dry vials, with one-half of the blood sample volume collected in a tube containing an anticoagulant (ethylenediaminetetraaceticacid 
[EDTA] for plasma separation and testing) and the other half collected in a tube without anticoagulant (for serum testing). This blood sample for plasma testing was centrifuged at $3000 \mathrm{rpm}$ for $10 \mathrm{~min}$, and the supernatant was used for determining the fasting glucose level. Fasting blood glucose (FBG) was measured by the glucose oxidase method. The blood specimen collected in the plain tube (without anticoagulant) was allowed to clot and then centrifuged at $3000 \mathrm{rpm}$ for $10 \mathrm{~min}$ for separation of the serum. An enzymatic colorimetric test method was used to measure the serum creatinine level using the fully automated HumaStar 200 clinical chemistry analyzer (Human Diagnostics [Human Biochemica und Diagnostica $\mathrm{GmbH}]$, Wiesbaden, Germany).

Serum creatinine was measured in all participants during the data collection period. The eGFR was calculated using the Modification of Diet in Renal Disease (MDRD) formula, and the results were categorized into normal/higher eGFR $\left(\geq 90 \mathrm{ml} / \mathrm{min} / 1.73 \mathrm{~m}^{2}\right)$ and reduced eGFR $\left(<90 \mathrm{ml} / \mathrm{min} / 1.73 \mathrm{~m}^{2}\right)$.

\section{Anthropometric Measurements}

Body weight was measured on an adult weighing scale to the nearest $0.5 \mathrm{~kg}$, with the patient wearing light clothes and in bare feet or stocking feet. Height was measured using a standard height board; the head piece of the height board was gradually lowered until it reached the patient's head and was at a $90^{\circ}$ angle with the measuring scale. The measurements were then taken to the nearest centimeter. BMI was calculated as weight in kilograms divided by height in meters squared.

\section{Data Quality Assurance}

Four data collectors and two supervisors were trained for 2 days. The questionnaire was back translated into English from local languages by a third person to check its consistency. The data collection tool was first pretested on 17 (5.0\%) patients with diabetes at the ambulatory clinic of Jimma University Medical Center to check the consistency, applicability and understandability of the questionnaire and abstraction format. Questions which were misunderstood were corrected for data collection. All completed data collection forms was checked each day for their completeness, consistency, clarity and accuracy by the principal investigators.

\section{Data Processing and Statistical Analysis}

Prior to data entry, data were coded and edited appropriately by the principal investigators. The data were then entered into Epidata Manager version 4.4.2, and following double entry verification the data were exported to SPSS version 24.0 (IBM Corp., Armonk, NY, USA) for analysis. Descriptive statistics, such as frequency, proportion, mean and standard deviation (SD), was used to describe glycemic control and the sociodemographic, clinical and behavioral characteristics of the patients. Bivariate logistic regression analysis was used to determine factors associated with poor glycemic control. Variables found to be significant at $p<0.25$ in the bivariate analysis were included in the multivariate logistic regression analysis to identify predictors of poor glycemic control. Statistical significance was set at $p<0.05$.

\section{Operational Definitions}

Glycemic Control Level. The glycemic control of each patient was categorized as good if the 3-month average FBS ranged from 80 to $130 \mathrm{mg} / \mathrm{dl}$; a value $>130 \mathrm{mg} / \mathrm{dl}$ was considered to indicate poor glycemic control [39]. FBS. FBS was measured after at least $8 \mathrm{~h}$ of fasting [39].

Diabetes knowledge. Participants who answered correctly to more than or equal to one-half of all questions on their diabetes knowledge were categorized as having a good knowledge of diabetes; correct answers to less than one-half of the questions indicted poor diabetes knowledge.

Diet Adherence. Patients with diabetes who reported an average of 4-7 days of information to the questions on diet were classified as practicing good dietary habits; those 
reporting $<4$ days were classified as having poor dietary practice.

Physical Activity. Patients with diabetes who participated in at least $30 \mathrm{~min}$ of physical activity daily or participated in a specific exercise session were considered to have adequate physical activity; otherwise, the patient was classified as having inadequate physical activity.

$S M B G$. Patients who performed SMBG at their respective home for $>3$ days during the last 7 days were considered to have performed SMBG.

Medication Adherence. Patients who took all the prescribed antidiabetic medications during the last 7 days were considered to be medication adherent.

Co-Morbidity. Patients with any chronic disease that coexisted with their diabetes were considered to be co-morbid [42].

Polypharmacy. Polypharmacy was defined as the use of $\geq 5$ medications [43].

BMI. BMI was classified as underweight $\left(<18.5 \mathrm{~kg} / \mathrm{m}^{2}\right), \quad$ normal weight (18.5-24.9 kg/m²), overweight (25.0-29.9 kg/ $\mathrm{m}^{2}$ ) or obese $\left(\geq 30.0 \mathrm{~kg} / \mathrm{m}^{2}\right)$ [44].

\section{Ethical Considerations}

The institutional review board of Jimma University approved the study (reference number IHRPGC/566/2019), and a Letter of Permission was forwarded to the administration of Mettu Karl referral hospital. The study was conducted in accordance with the Helsinki Declaration of 1964, and its later amendments. Each patient provided written informed consent for the use of personal and clinical data. All data collected were treated with strict confidentiality at all stages of data processing.

\section{RESULTS}

\section{Socio-Demographic Characteristics of the Study Participants}

A total of 330 patients with diabetes participated in this study, of whom 198 were male.
The mean age of the participants was $49.85 \pm 14.19$ years, and $156(47.3 \%)$ were between 41 and 60 years of age. About 114 $(34.5 \%)$ of participants had attained primary school level education. The most prevalent occupation among the participants was farmer (113; $34.2 \%)$; more than half $(53.6 \%)$ of participants were urban residents. About 154 (46.7\%) of participants had a monthly income $>1000$ Ethiopian Birr (ETB). The mean $( \pm$ SD) BMI of the participants was determined to be $23.89 \pm 4.27 \mathrm{~kg} / \mathrm{m}^{2}$, and $63.6 \%$ had a healthy weight (Table 1 ).

\section{Diabetes Self-Care Practice and Knowledge of Diabetes of Study Participants}

Almost all participants (96.1\%) had never smoked cigarettes. Regarding alcohol consumption, $18.5 \%$ had a history of consuming alcohol but no longer did so, and $7.9 \%$ of participants currently consumed alcohol. The majority $(82.4 \%)$ of study participants did not chew chat. One hundred and thirty-one (39.7\%) participants reported following a sufficiently healthy eating plan during the week preceding the study period. Assessment of the level of physical activity of the participants revealed that $40.3 \%$ performed at least $30 \mathrm{~min}$ of physical activity daily during the 7 days immediately preceding the study period. The prevalence of non-adherence to antidiabetes medications was $59.1 \%, 52.4 \%$ of participants had a good knowledge of diabetes and about $22.4 \%$ of participants were adhering to monitoring their blood sugar at home (Table 2).

\section{Clinical Characteristics of Study Participants}

The mean $( \pm$ SD) duration of diabetes among the study participants was $7.72 \pm 5.91$ years, with $30.9 \%$ having a disease duration of $\geq 10$ years. Assessment of the family history of diabetes revealed that $28.8 \%$ of participants had a positive family history of diabetes . Ninety-eight (29.7\%) study participants had a history of diabetes ketoacidosis. About $43.3 \%$ of study participants had additional chronic disease(s), 
Table 1 Socio-demographic characteristics of participants in the study conducted at Mettu Karl referral hospital in 2019

\begin{tabular}{|c|c|c|c|c|}
\hline Variables & Categories & Type 1 DM, $n(\%)$ & Type 2 DM, $n(\%)$ & Total, $n(\%)$ \\
\hline \multirow[t]{2}{*}{ Sex } & Male & $87(43.9)$ & $111(56.1)$ & $198(60.0)$ \\
\hline & Female & $41(31.1)$ & $91(68.9)$ & $132(40.0)$ \\
\hline \multirow[t]{3}{*}{ Age (years) } & $18-40$ & $70(72.9)$ & $26(27.1)$ & $96(29.1)$ \\
\hline & $41-60$ & $45(28.8)$ & $111(71.2)$ & $156(47.3)$ \\
\hline & $>60$ & $13(16.7)$ & $65(83.3)$ & $78(23.6)$ \\
\hline \multirow[t]{4}{*}{ Educational status } & No formal education & $31(43.7)$ & $40(56.3)$ & $71(21.5)$ \\
\hline & Primary education (grades 1-8) & $47(41.2)$ & $67(58.8)$ & $114(34.5)$ \\
\hline & Secondary education (grades 9-12) & $32(49.2)$ & $33(50.8)$ & $65(19.7)$ \\
\hline & $\begin{array}{l}\text { Tertiary education (college/ } \\
\text { university) }\end{array}$ & $18(22.5)$ & $62(77.5)$ & $80(24.2)$ \\
\hline \multirow[t]{6}{*}{ Occupation } & Farmers & $55(48.7)$ & $58(51.3)$ & $113(34.2)$ \\
\hline & Merchants & $43(44.3)$ & $54(55.7)$ & $97(29.4)$ \\
\hline & Employees & $12(26.7)$ & $33(73.3)$ & $45(13.6)$ \\
\hline & Housewives & $6(14.6)$ & $35(85.4)$ & $41(12.4)$ \\
\hline & Retired & $5(20.0)$ & $20(80.0)$ & $25(7.6)$ \\
\hline & Others $^{\mathrm{a}}$ & $7(77.8)$ & $2(22.2)$ & $9(2.7)$ \\
\hline \multirow[t]{2}{*}{ Residence } & Urban & $55(31.1)$ & $122(68.9)$ & $177(53.6)$ \\
\hline & Rural & $73(47.7)$ & $80(52.3)$ & $153(46.4)$ \\
\hline \multirow[t]{3}{*}{ Monthly income (ETB) } & $<500$ & $20(51.3)$ & $19(48.7)$ & $39(11.8)$ \\
\hline & $500-1000$ & $56(40.9)$ & $81(59.1)$ & $137(41.5)$ \\
\hline & $>1000$ & $52(33.8)$ & $102(66.2)$ & $154(46.7)$ \\
\hline \multirow[t]{4}{*}{$\operatorname{BMI}\left(\mathrm{kg} / \mathrm{m}^{2}\right)$} & Underweight $(<18.5)$ & $4(21.1)$ & $15(78.9)$ & $19(5.8)$ \\
\hline & Healthy weight (18.5-24.9) & $106(50.5)$ & $104(49.5)$ & $210(63.6)$ \\
\hline & Overweight (25-29.9) & $11(18.6)$ & $48(81.4)$ & $59(17.9)$ \\
\hline & Obese $(\geq 30)$ & $7(16.7)$ & $35(83.3)$ & $42(12.7)$ \\
\hline
\end{tabular}

Values in table are presented as the number of patients (frequency) with the percentage in parenthesis $B M I$ Body mass index, DM diabetes mellitus, ETB Ethiopian Birr

${ }^{a}$ Daily laborers, drivers and students

with hypertension being the most common comorbidity (39.1\% of study participants). The eGFR was $\geq 90 \mathrm{ml} / \mathrm{min}$ per $1.73 \mathrm{~m}^{2}$ in 196 (59.4\%) participants (Table 3).

\section{Patterns of Medication Use Among Study Participants}

Oral antidiabetes drugs were prescribed to $45.5 \%$ of the patients, of whom $27.6 \%$ were on a combination therapy of metformin + 
Table 2 Diabetes self-care activities and knowledge of participants in the study conducted at Mettu Karl referral hospital in 2019

\begin{tabular}{llccc}
\hline Variables & Categories & Type 1 DM, $\boldsymbol{n}$ (\%) & Type 2 DM, $\boldsymbol{n}$ (\%) & Total, $\boldsymbol{n}$ (\%) \\
\hline Smoking status & Never smoked & $124(39.1)$ & $193(60.9)$ & $317(96.1)$ \\
& Yes, previously & $4(33.3)$ & $8(66.7)$ & $12(3.6)$ \\
& Current smoker & $0(0.0)$ & $1(100.0)$ & $1(0.3)$ \\
& No, never & $89(36.6)$ & $154(63.4)$ & $243(73.6)$ \\
Alcohol consumption & Yes, previously & $31(50.8)$ & $30(49.2)$ & $61(18.5)$ \\
& Yes, currently & $8(30.8)$ & $18(69.2)$ & $26(7.9)$ \\
Chewing chat & No, never & $105(38.6)$ & $167(61.4)$ & $272(82.4)$ \\
& Yes, previously & $13(39.4)$ & $20(60.6)$ & $33(10.0)$ \\
& Yes, currently & $10(40.0)$ & $15(60.0)$ & $25(7.6)$ \\
Diet adherence & Good & $46(35.1)$ & $85(64.9)$ & $131(39.7)$ \\
Physical activity & Poor & $82(41.2)$ & $117(58.8)$ & $199(60.3)$ \\
& Adequate & $51(38.3)$ & $82(61.7)$ & $133(40.3)$ \\
Drug adherence & Inadequate & $77(39.1)$ & $120(60.9)$ & $197(59.7)$ \\
Knowledge of optimum blood sugar & Yes & $50(37.0)$ & $85(63.0)$ & $135(40.9)$ \\
& Yes & $78(40.0)$ & $117(60.0)$ & $195(59.1)$ \\
Adtatus of diabetes knowledge & No & $91(39.8)$ & $56(60.2)$ & $93(28.2)$ \\
& Yes & $23(31.1)$ & $146(61.6)$ & $237(71.8)$ \\
& No & $105(41.0)$ & $151(59.0)$ & $74(22.4)$ \\
& Good & $56(32.4)$ & $117(67.6)$ & $173(52.4)$ \\
& Poor & $72(45.9)$ & $85(54.1)$ & $157(47.6)$ \\
& No & $59(45.4)$ & $71(54.6)$ & $130(39.4)$ \\
& $69(34.5)$ & $131(65.5)$ & $200(60.6)$ \\
\hline
\end{tabular}

Values in table are presented as the number of patients (frequency) with the percentage in parenthesis $B S$ Blood sugar, $S M B G$ self-monitoring of blood glucose

glibenclamide. Insulin injection was prescribed to $40.3 \%$ of the patients. Polypharmacy was prescribed to $6.7 \%$ of study participants. Statin therapy and aspirin therapy were initiated for 12.1 and $10.0 \%$ of participants, respectively (Table 4).

\section{Glycemic Control Among Study Participants}

The mean $( \pm \mathrm{SD})$ baseline FBS level was $188.28 \pm 75.69 \mathrm{mg} / \mathrm{dl}$ and the mean 3-month average FBS was $178.34 \pm 53.73 \mathrm{mg} / \mathrm{dl}$. The 3-month average FBS measurement indicated that $72.7 \%$ of study participants had poor glycemic control (Table 5). 
Table 3 Clinical characteristics of participants in the study conducted at Mettu Karl referral hospital in 2019

\begin{tabular}{|c|c|c|c|c|}
\hline Variables & Categories & Type 1 DM, $n(\%)$ & Type 2 DM, $n(\%)$ & Total, $n(\%)$ \\
\hline \multirow[t]{2}{*}{ Duration of DM (years) } & $<10$ & $82(36.0)$ & $146(64.0)$ & $228(69.1)$ \\
\hline & $\geq 10$ & $46(45.1)$ & $56(54.9)$ & $102(30.9)$ \\
\hline \multirow[t]{2}{*}{ Family history of DM } & Yes & $20(21.1)$ & $75(78.9)$ & $95(28.8)$ \\
\hline & No & $108(46.0)$ & $127(54.0)$ & $235(71.2)$ \\
\hline \multirow[t]{2}{*}{ History of DKA } & Yes & $70(71.4)$ & $28(28.6)$ & $98(29.7)$ \\
\hline & No & $58(25.0)$ & $174(75.0)$ & $232(70.3)$ \\
\hline \multirow[t]{2}{*}{ Presence of comorbidities } & Yes & $48(33.6)$ & $95(66.4)$ & $143(43.3)$ \\
\hline & No & $80(42.8)$ & $107(57.2)$ & $187(56.7)$ \\
\hline \multirow[t]{5}{*}{ Type of comorbidities } & Hypertension & $43(33.3)$ & $86(66.7)$ & $129(39.1)$ \\
\hline & Heart failure & $4(20.0)$ & $16(80.0)$ & $20(6.1)$ \\
\hline & Asthma & $4(50.0)$ & $4(50.0)$ & $8(2.4)$ \\
\hline & Ischemic heart disease & $0(0.0)$ & $7(100.0)$ & $7(2.1)$ \\
\hline & Others $^{a}$ & $2(25.0)$ & $6(75.0)$ & $8(2.4)$ \\
\hline \multirow[t]{2}{*}{ Presence of complications } & Yes & $35(27.6)$ & $92(72.4)$ & $127(38.5)$ \\
\hline & No & $93(45.8)$ & $110(54.2)$ & $203(61.5)$ \\
\hline \multirow[t]{4}{*}{ Type of complications } & Neuropathy & $14(17.7)$ & $65(82.3)$ & $79(23.9)$ \\
\hline & Retinopathy & $16(39.0)$ & $25(61.0)$ & $41(12.4)$ \\
\hline & Nephropathy & $6(18.8)$ & $26(81.2)$ & $32(9.7)$ \\
\hline & Others $^{\mathrm{b}}$ & $1(16.7)$ & $5(83.3)$ & $6(1.8)$ \\
\hline \multirow[t]{2}{*}{ Serum Creatinine $(\mathrm{mg} / \mathrm{dl})$} & Normal & $114(39.4)$ & $175(60.6)$ & $289(87.6)$ \\
\hline & Increased & $14(34.1)$ & $27(65.9)$ & $41(12.4)$ \\
\hline \multirow[t]{2}{*}{ eGFR $\left(\mathrm{ml} / \mathrm{min}\right.$ per $\left.1.73 \mathrm{~m}^{2}\right)$} & $\geq 90$ & $88(44.9)$ & $108(55.1)$ & $196(59.4)$ \\
\hline & $<90$ & $40(29.9)$ & $94(70.1)$ & $134(40.6)$ \\
\hline
\end{tabular}

Values in table are presented as the number of patients (frequency) with the percentage in parenthesis

$D K A$ Diabetes ketoacidosis, $D M$ diabetes mellitus, $e G F R$ estimated glomerular filtration rate

a Stroke, toxic goiter and human immunodeficiency virus (HIV)

b Impotency and foot ulcer

\section{Factors Associated with Poor Glycemic Control}

The association between independent variables and the dependent variable, glycemic control status, was investigated using both univariate and multivariate logistic regression. Univariate logistic regression analysis revealed that factors associated with poor glycemic control included age 18-40 years [crude odds ratio (COR) 1.79; $95 \%$ CI $0.90,3.56 ; p=0.095]$, no formal education (COR 4.24; 95\% CI 1.78, 10.09; $p=0.001)$, duration of diabetes $\geq 10$ years (COR 2.36; 95\% CI 1.31, 4.25; $p=0.004$ ), overweight (COR 2.66; 95\% CI 1.24, 5.73; $p=0.012$ ), obesity (COR 2.39; 95\% CI 1.01, 
Table 4 Patterns of medication use among participants in the study conducted at Mettu Karl referral hospital in 2019

\begin{tabular}{lccc}
\hline Variables & Type 1 DM, $\boldsymbol{n}(\%)$ & Type 2 DM, $\boldsymbol{n}$ (\%) & Total, $\boldsymbol{n}(\%)$ \\
\hline Insulin alone & $107(80.5)$ & $26(19.5)$ & $133(40.3)$ \\
Metformin alone & $3(6.0)$ & $47(94.0)$ & $50(15.2)$ \\
Glibenclamide alone & $0(0.0)$ & $9(100.0)$ & $9(2.7)$ \\
Insulin + metformin combination therapy & $18(38.3)$ & $29(61.7)$ & $47(14.2)$ \\
Metformin + glibenclamide combination therapy & $1(1.1)$ & $90(98.9)$ & $91(27.6)$ \\
Polypharmacy & & & \\
Yes & $4(18.2)$ & $18(81.8)$ & $308(93.3)$ \\
No & $124(40.3)$ & $184(59.7)$ & \\
Statin therapy & & & $40(12.1)$ \\
Yes & $4(10.0)$ & $36(90.0)$ & $290(87.9)$ \\
No & $124(42.8)$ & $166(57.2)$ & $33(10.0)$ \\
Aspirin therapy & & $30(90.9)$ & $297(90.0)$ \\
Yes & $3(9.1)$ & $172(57.9)$ & \\
No & $125(42.1)$ &
\end{tabular}

Values in table are presented as the number of patients (frequency) with the percentage in parenthesis

Table 5 Fasting blood sugar measurements among participants in the study conducted at Mettu Karl referral hospital in 2019

\begin{tabular}{|c|c|c|c|}
\hline \multirow[t]{2}{*}{ FBS measurements } & \multirow[t]{2}{*}{ Mean \pm SD } & \multicolumn{2}{|l|}{ Glycemic control status } \\
\hline & & Good $(80-130 \mathrm{mg} / \mathrm{dl})$ & Poor $(>130 \mathrm{mg} / \mathrm{dl})$ \\
\hline Baseline & $188.28 \pm 75.69$ & $85(25.8)$ & $245(74.2)$ \\
\hline Month-1 & $184.10 \pm 77.20$ & $103(31.2)$ & $227(68.8)$ \\
\hline Month-2 & $176.08 \pm 68.92$ & $101(30.6)$ & $229(69.4)$ \\
\hline Month-3 & $174.85 \pm 63.36$ & $98(29.7)$ & $232(70.3)$ \\
\hline Three-month average & $178.34 \pm 53.73$ & $90(27.3)$ & $240(72.7)$ \\
\hline
\end{tabular}

FBS measurements are presented as the measure or 3-month average value (in $\mathrm{mg} / \mathrm{dl}$ ) with the standard deviation given in parenthesis

FBS Fasting blood sugar

$5.67 ; p=0.047$ ), higher eGFR (COR 2.05; 95\% CI $1.25,3.34 ; p=0.004)$, poor diet adherence (COR 8.68; 95\% CI 4.96, 15.21; $p<0.001$ ), physical inactivity (COR 1.73; 95\% CI 1.06, $2.82 ; p=0.029)$, non- adherence to medications (COR 6.98; 95\% CI 3.86, 12.62; $p<0.001$ ], poor knowledge of optimum blood sugar level (COR
$3.73 ; 95 \%$ CI 2.22, 6.27; $p<0.001)$, poor knowledge of diabetes (COR 1.85; 95\% CI 1.12, $3.04 ; p=0.016)$, type 1 diabetes (COR $2.10 ; 95 \%$ CI $1.24,3.58 ; p=0.006)$ and insulin therapy (COR 1.69; 95\% CI 0.99, 2.88; $p=0.056$ ).

All factors associated with $p$ value of $<0.25$ on the univariate analysis were included in the 
multivariate logistic regression analysis, which revealed that overweight, obesity, higher eGFR, poor diet adherence, non-adherence to medications and type 1 diabetics were significantly associated with poor glycemic control. Compared to patients with a normal healthy weight, poor glycemic control were 4.07-fold more likely in overweight patients [adusted odds ratio (AOR) 4.07; 95\% CI 1.60, 10.36; $p=0.003$ ] and 4.39-fold more likely in obese patients (AOR: 4.39 ; 95\% CI 1.59, $12.14 ; p=0.004)$. The likelihood of poor glycemic control was about 2.34fold more likely among participants who had an eGFR $\geq 90 \mathrm{ml} / \mathrm{min} / 1.73 \mathrm{~m}^{2}$ when compared to those who had an GFR $<90 \mathrm{ml} / \mathrm{min} / 1.73 \mathrm{~m}^{2}$ (AOR 2.34; 95\% CI 1.23, 4.44; $p=0.010$ ). Participants with type 1 diabetics were 3.22 -fold more likely to have poorly controlled blood sugar as compared to those with type 2 diabetics (AOR 3.22; 95\% CI 1.58, 6.55; $p=0.001$ ). The likelihood of poor glycemic control was almost sevenfold more likely among participants who did not adhere to the recommended diet than among those you did (AOR: 6.95; 95\% CI 3.63, 13.32); $p<0.001$ ) and 5.82-fold more likely among those who did not adhere to their antidiabetes medication than among those you did adhere to their therapeutic regimen (AOR 5.82, 95\% CI 2.77, 12.26; $p<0.001$ ) (Table 6).

\section{DISCUSSION}

Glycemic control plays a key role in preventing diabetes-related complications. Persons with uncontrolled diabetics are at a much higher risk of complications, such as retinopathy, nephropathy, neuropathy and cardiovascular disease. The adequacy of glycemic control in diabetes is also important in reducing morbidity and mortality of the disease $[24,45,46]$. The most effective method to avoid or delay these complications and to prevent target organ damage is to achieve the glycemic target [23].

In this study we assessed glycemic control level and its predictors among diabetic patients in Ethiopia. The proportion of patients with poor glycemic control in our study population was $72.7 \%$, which is comparable to results reported from studies in northeast Ethiopia
(70.8\% with poor glycemic control [32]), in Uganda $(73.5 \%$ with poor glycemic control [47]) and in Brazil (76.0\% with inadequate glycemic control [48]). However, this result was higher than the $59.4 \%$ reported from a study in Jimma, southwest Ethiopia [30], 61.9\% from a study in Mekele, north Ethiopia [34], 64.7\% from a study in Gondar, north Ethiopia [33] and $60.5 \%$ from a study in Kenya [49], with all of these studies also including patients with type 1 and 2 diabetes. This discrepancy may be due to the use of HbA1c in all these studies to assess glycemic control. Alternatively, differences in culture, environmental factors and genetics may have affected glycemic control.

We observed that patients with poorly controlled blood glucose were more likely to be overweight or obese patients when compared to those with a healthy weight. This result correlates with a study in the USA which reported a statistically significant association between being overweight or obese and poor glycemic control [22]. Studies in Saudi Arabia and India have also demonstrated that overweight and obesity are determinants of poor glycemic control $[10,50]$. One explanation for this effect of overweight or obesity is the consumption of food with a high content of carbohydrates and high glycemic index, which increases fat storage and makes it more difficult for persons with diabetes to their control blood sugar level. Excessive storage of fat and high glucose levels due to carbohydrate overconsumption contribute to poor glycemic control [26].

The findings of this study show that patients with diabetes with a higher eGFR were more likely to have poorly controlled blood glucose than those who had a reduced eGFR. This result is consistent with those reported from a number of earlier studies. In one study conducted in the USA, the authors reported that higher eGFR was related to poor glycemic control [51]. Tsuda et al. suggested that their results indicated that serum creatinine levels are lower in diabetic patients than in their nondiabetic counterparts, possibly leading to a higher eGFR in the former. They also suggested that sustained elevations of plasma glucose might lead to increased eGFR in patients with diabetes [52], and Naderpoor et al. reported that that higher eGFR has been 


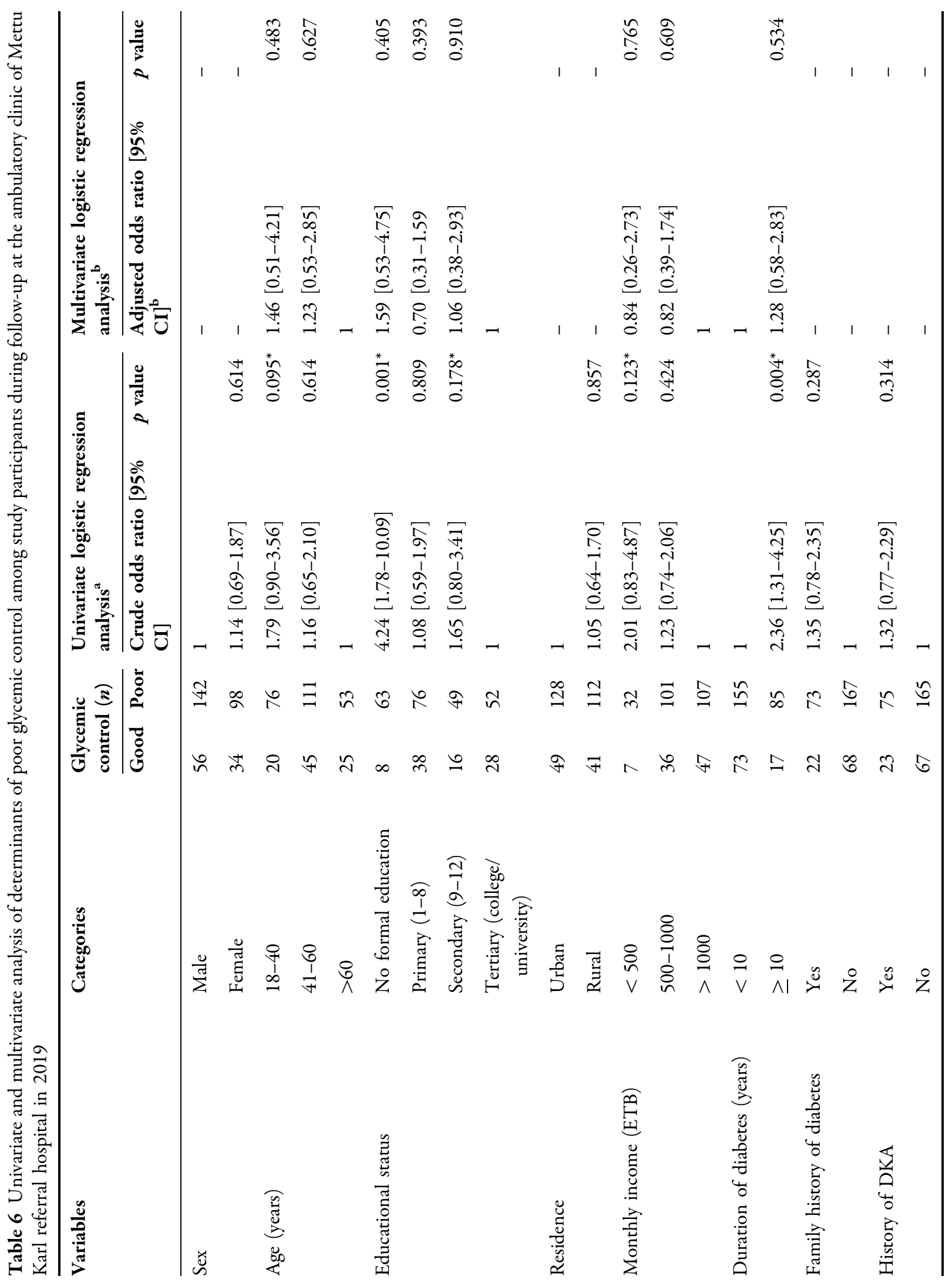




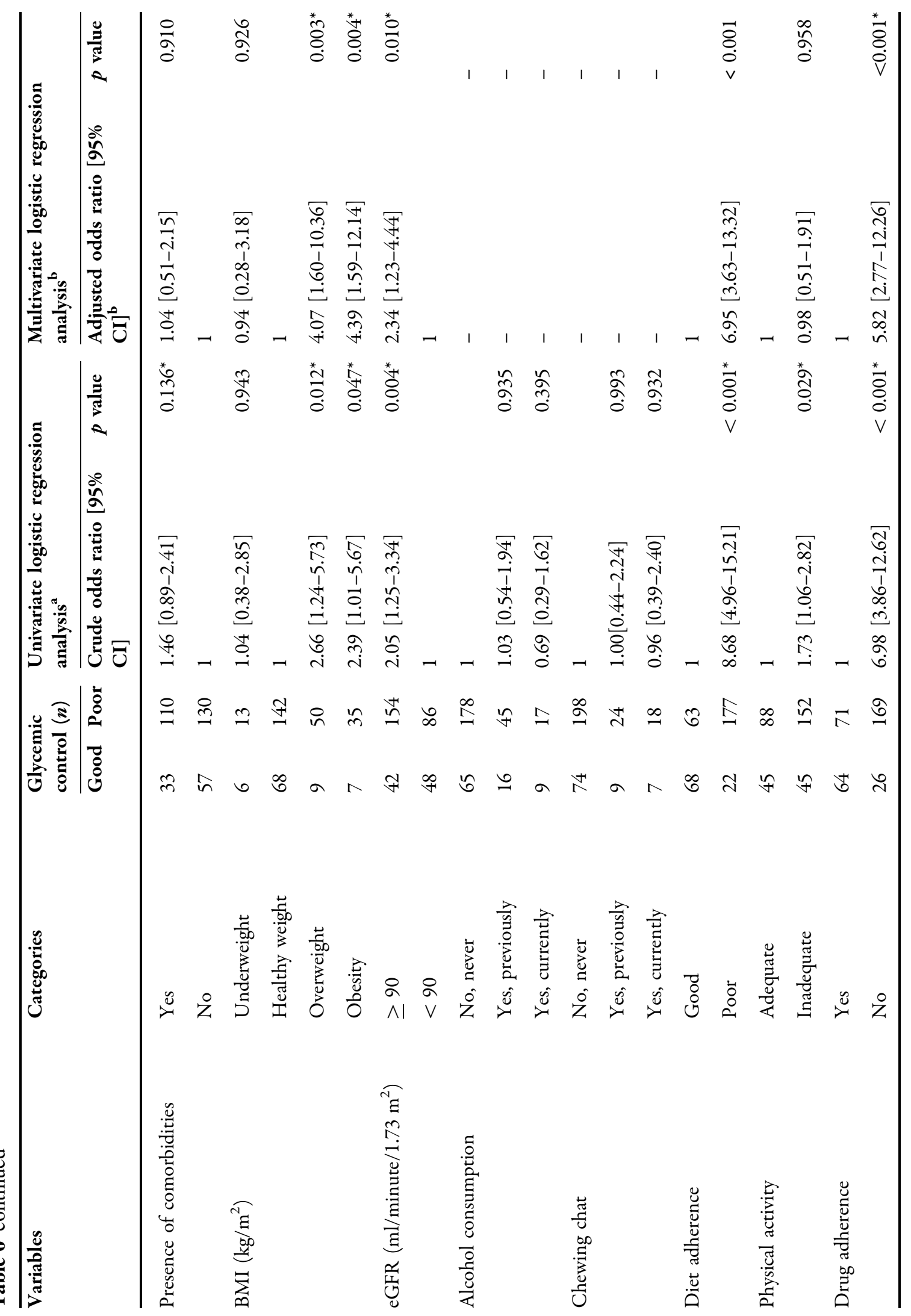




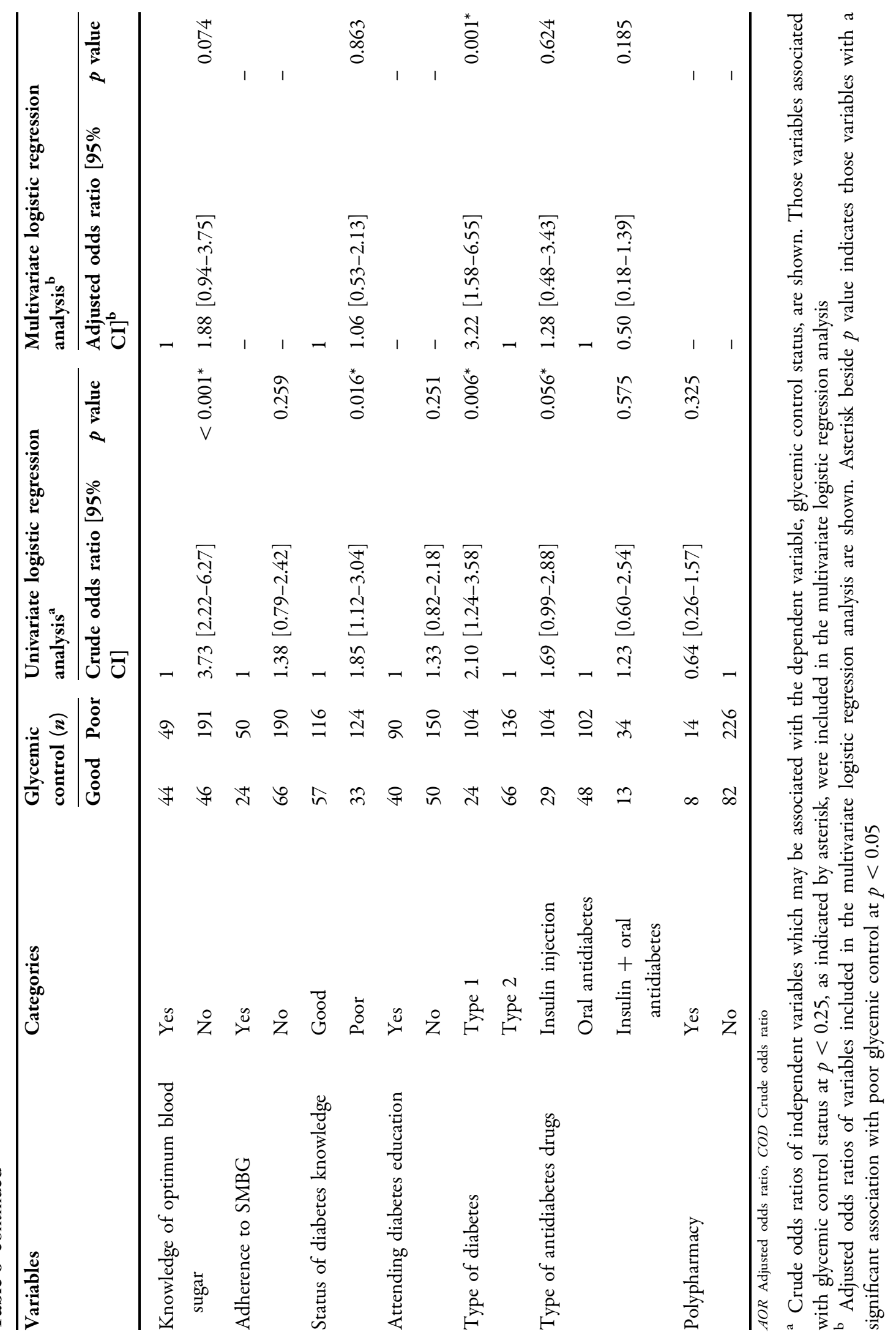


associated with insulin resistance, even in healthy individuals [53].

Our analyses determined that those participants who did not adhere to their recommended diet were more likely to have poorly controlled blood glucose than participants who did adhere to the recommended diet. Consistent with this result, a study in Gulf Cooperation Council Countries demonstrated that poor compliance to diet control is significantly associated with poor glycemic control [19]. Studies conducted in India and Turkey have also reported that poor compliance to diet recommendation contributes to poor glycemic control [37, 46]. It is obvious that lifestyle modifications, such as compliance to the recommended diet, are very important strategies for controlling patients' blood sugar. Thus, poor adherence to a recommended diet might make it more difficult to control blood sugar.

There was a significant association between medication adherence and poor glycemic control. Participants who failed to adhere to their prescribed medications were more likely to have poor glycemic control. This finding is similar to those from previous studies, including one conducted in Ethiopia (Gondar and Jimma) $[33,35]$ and another conducted in Tanzania, with the latter demonstrating that patients with poor adherence to prescribed antidiabetes medications had significantly poor glycemic control [25]. Adherence to prescribed antidiabetic medications is crucial to reach glycemic control as non-adherence with blood glucoselowering drugs is associated with higher HbA1c [54]. Therefore, poor glycemic control could be a result of poor adherence to antidiabetes medications.

According to our study results, the proportion of patients with poor glycemic control was higher among those with type 1 diabetes than among those with type 2 diabetes. This finding is similar to results from a study conducted in Brazil which reported that the rates of inadequate glycemic control were higher in patients with type 1 diabetes than in patients with type 2 diabetes [48]. A study conducted in Gondar, Ethiopia also observed that glycemic control was less likely to be controlled in patients with type 1 diabetes [33]. One possible explanation for the poorer glycemic control in patients with type 1 diabetes as compared to those with type 2 diabetes might be that the absolute deficiency of insulin which causes type 1 diabetes mellatus may result in the body being less responsive to externally administered insulin. Another explanations may be that type 1 diabetes may be the consequence of inappropriate storage of insulin, measuring an incorrect dose of insulin, inappropriate insulin injection technique and inappropriate site of administration.

We observed that the age of the participants in our study was not significantly associated with glycemic control in the multivariate analysis. This finding is inconsistent with those reported from studies carried out in Iran and Saudi Arabia, both of which reported that glycemic control is significantly associated with age and that poor glycemic control is more likely with increasing age $[27,28]$. One explanation for the results of these latter studies is that the severity of diabetes increases over time; as such, it will be more difficult to control glycemic level in later life when this was not achieved early after diagnosis [26]. In contrast, a number of other studies have indicated that a younger age is associated with poor glycemic control, as shown in studies conducted in Saudi Arabia [24], Malaysia [26] and Japan [29], all of which showed that glycemic control was more difficult among younger ages [26].

We also found that there was no relationship between the gender of the study participants and glycemic control. In contrast, a study conducted by Harrabi et al. [24] in Saudi Arabia reported that being female was associated with poor glycemic control while a study by Demoz et al. [55] in Ethiopia also reported that being female was significantly associated with poor glycemic control. One possible reason for this gender difference may be that it is the female who is usually the caregiver for the entire family (including not only the husband and children but also mother and mother-in-law), which might increase her domestic responsibilities. However, a study done by Mahmood et al. [26] in Malaysia found that being male associated with poor glycemic control. The finding in this latter study may be due to local and regional phenomena as most other studies have found 
that gender is not associated with glycemic control [24].

One of the limitations of this study was the use of FBS to assess glycemic control level since the HbA1c test was not available in the study area. Measurements of FBG levels provide a short-term picture of control. Measurement of HbA1c is now universally accepted as the most reliable indicator of long-term glycemic control because it accurately reflects an individual's blood glucose levels over the preceding 2-3 months [37]. Another limitation of our study was that data on such variables as medication adherence, physical exercise and diet adherence were obtained by self-report and may be limited by recall bias.

\section{CONCLUSION}

The results of this study indicate that the patients with diabetes in Mettu Karl referral hospital generally have poor glycemic control. Almost three-quarters of the study participants had poorly controlled blood sugar. We also demonstrated that poor glycemic control was significantly associated with overweight, obesity, higher eGFR, poor diet adherence, nonadherence to medications and type 1 diabetics. Based on these results, we suggest that the educational approaches provided by healthcare providers should focus on enhancing measures focused on improving dietary control, drug adherence and weight control and optimizing glycemic level. Healthcare providers should clearly focus on the importance of good glycemic control so that patients can be protected from the complications of diabetes and potentially avoidable glycemic burden.

\section{ACKNOWLEDGEMENTS}

We would like to thank Jimma University for supporting the study. We are very grateful to the staff of Mettu Karl referral hospital, study participants, data collectors and supervisors for their cooperation that enable the successful completion of this study.
Funding. This study was sponsored by Jimma University. The funding body played no any role in study design, data collection, analysis and interpretation of data and writing of the manuscript or in the decision to submit the manuscript for publication. No funding or sponsorship was received for the publication of this article.

Authorship. All named authors meet the International Committee of Medical Journal Editors (ICMJE) criteria for authorship for this article, take responsibility for the integrity of the work as a whole, and have given their approval for this version to be published.

Disclosures. Tadesse Sheleme, Girma Mamo, Tsegaye Melaku and Tamiru Sahilu have nothing to disclose.

Compliance with Ethics Guidelines. The institutional review board of Jimma University approved the study (reference number IHRPGC/ 566/2019), and a Letter of Permission was forwarded to the administration of Mettu Karl referral hospital. The study was conducted in accordance with the Helsinki Declaration of 1964, and its later amendments. Each patient provided written informed consent for the use of personal and clinical data. All data collected were treated with strict confidentiality at all stages of data processing.

Data Availability. The datasets generated during and/or analyzed during the current study are available from the corresponding author on reasonable request.

Open Access. This article is licensed under a Creative Commons Attribution-NonCommercial 4.0 International License, which permits any non-commercial use, sharing, adaptation, distribution and reproduction in any medium or format, as long as you give appropriate credit to the original author(s) and the source, provide a link to the Creative Commons licence, and indicate if changes were made. The images or other third party material in this article are included in the article's Creative Commons licence, unless indicated otherwise in a credit 
line to the material. If material is not included in the article's Creative Commons licence and your intended use is not permitted by statutory regulation or exceeds the permitted use, you will need to obtain permission directly from the copyright holder. To view a copy of this licence, visit http://creativecommons.org/licenses/by$\mathrm{nc} / 4.0 /$.

\section{REFERENCES}

1. Al-Akour N, Khader Y, Alaoui A. Glycemic control and its determinants among patients with type 2 diabetes mellitus attending a teaching hospital. J Diabetes Metab. 2011;2:129.

2. World Health Organization. Global report on diabetes: World Health Organization:Geneva; 2016. http://apps.who.int/iris/bitstream/10665/204871/ 1/9789241565257_eng.pdf. Accessed 15 Jan 2019.

3. Saeedi P, Petersohn I, Salpea P, et al. Global and regional diabetes prevalence estimates for 2019 and projections for 2030 and 2045: results from the International Diabetes Federation Diabetes Atlas, 9th edition. Diabetes Res Clin Pract. 2019;157(107843):10.

4. Ayele K, Tesfa B, Abebe L, Tilahun T, Girma E. Self care behavior among patients with diabetes in Harari, Eastern Ethiopia: the health belief model perspective. PLoS One. 2012;7(4):e35515.

5. Stanifer JW, Cleland CR, Makuka GJ, et al. Prevalence, risk factors, and complications of diabetes in the Kilimanjaro region: a population-based study from Tanzania. PLoS One. 2016;11(10):e0164428.

6. Gebreyohannes EA, Netere AK, Belachew SA. Glycemic control among diabetic patients in Ethiopia: a systematic review and meta-analysis. PLoS One. 2019;14(8):e0221790.

7. Baena-Díez JM, Peñafiel J, Subirana I, et al. Risk of cause-specific death in individuals with diabetes: a competing risks analysis. Diabetes Care. 2016;39(11):1987-95.

8. Cho NH, Shaw JE, Karuranga S, et al. IDF Diabetes Atlas: global estimates of diabetes prevalence for 2017 and projections for 2045. Diabetes Res Clin Pract. 2018;138:271-81.

9. Misganaw A, Mariam DH, Araya T. The double mortality burden among adults in Addis Ababa, Ethiopia, 2006-2009. Prev Chronic Dis. 2012;9:E84.
10. Alzaheb RA, Altemani AH. The prevalence and determinants of poor glycemic control among adults with type 2 diabetes mellitus in Saudi Arabia. Diabetes Metab Syndr Obes Targets Ther. 2018;11: 15.

11. Camara A, Baldé NM, Sobngwi-Tambekou J, et al. Poor glycemic control in type 2 diabetes in the South of the Sahara: the issue of limited access to an HbA1c test. Diabetes Res Clin Pract. 2015;108(1): 187-92.

12. Jaspinder Kaur SS, Kaur K. Determinants of the glycemic status and its relationship with cardiovascular risk factors. J Biochem Pharmacol Res. 2014;2(3):159-66.

13. Navarro-Pérez J, Orozco-Beltran D, Gil-Guillen V, et al. Mortality and cardiovascular disease burden of uncontrolled diabetes in a registry-based cohort: the ESCARVAL-risk study. BMC Cardiovasc Disord. 2018;18(1):180.

14. Pastakia SD, Pekny CR, Manyara SM, Fischer L. Diabetes in sub-Saharan Africa-from policy to practice to progress: targeting the existing gaps for future care for diabetes. Diabetes Metab Syndr Obes. 2017;10:247-63.

15. Fasil A, Biadgo B, Abebe M. Glycemic control and diabetes complications among diabetes mellitus patients attending at University of Gondar Hospital, Northwest Ethiopia. Diabetes Metab Syndr Obes Targets Ther. 2019;12:75.

16. Ramanathan RS. Correlation of duration hypertension and glycemic control with microvascular complications of diabetes mellitus at a tertiary care hospital. J Neurol Exp Neural Sci. 2017;4(1):1-4.

17. Fowler MJ. Microvascular and macrovascular complications of diabetes. Clin Diabetes. 2011;29(3): 116-22.

18. Tripathi BK, Srivastava AK. Diabetes mellitus: complications and therapeutics. Med Sci Monit. 2006;12(7):RA130-47.

19. Alramadan MJ, Afroz A, Hussain SM, et al. Patientrelated determinants of glycaemic control in people with type 2 diabetes in the Gulf Cooperation Council Countries: a systematic review. J Diabetes Res. 2018;2018:9389265.

20. Adeniyi OV, Yogeswaran P, Longo-Mbenza B, Ter Goon D, Ajayi AI. Cross-sectional study of patients with type 2 diabetes in OR Tambo district, South Africa. BMJ Open. 2016;6(7):e010875.

21. Adeniyi OV, Yogeswaran P, Wright G, LongoMbenza B. Diabetic patients' perspectives on the 
challenges of glycaemic control. Afr J Prim Health Care Fam Med. 2015;7(1):1-8.

22. Bae J, Lage M, Mo D, Nelson D, Hoogwerf B. Obesity and glycemic control in patients with diabetes mellitus: analysis of physician electronic health records in the US from 2009-2011. J Diabetes Complic. 2016;30(2):212-20.

23. AL-Eitan LN, Nassar AM, Saadeh NA, Almomani BA. Evaluation of glycemic control, lifestyle and clinical characteristics in patients with type 2 diabetes treated at King Abdullah university hospital in Jordan. Can J Diabetes. 2016;40(6):496-502.

24. Harrabi I, Al Harbi F, Al Ghamdi S. Predictors of glycemic control among patients with type 2 diabetes in Najran Armed Forces Hospital: a pilot study. J Diabetes Mellitus. 2014;4(02):141.

25. Kamuhabwa AR, Charles E. Predictors of poor glycemic control in type 2 diabetic patients attending public hospitals in Dar es Salaam. Drug Healthe Patient Saf. 2014;6:155.

26. Mahmood M, Daud F, Ismail A. Glycaemic control and associated factors among patients with diabetes at public health clinics in Johor, Malaysia. Public Health. 2016;135:56-65.

27. Maddah M, Attarpour L. Glycemic control and its predictors among Iranian diabetic patients. Mediterr J Nutr Metab. 2016;9(1):1-6.

28. Almutairi MA, Said SM, Zainuddin H. Predictors of poor glycemic control among type two diabetic patients. Am J Med Med Sci. 2013;3(2):17-21.

29. Tokuyama Y, Ishizuka T, Matsui K, Egashira T, Kanatsuka A. Predictors of glycemic control in Japanese subjects with type 2 diabetes mellitus. Metabolism. 2008;57(4):453-7.

30. Cheneke W, Suleman S, Yemane T, Abebe G. Assessment of glycemic control using glycated hemoglobin among diabetic patients in Jimma University specialized hospital, Ethiopia. BMC Res Notes. 2016;9(1):96.

31. Tekalegn Y, Addissie A, Kebede T, Ayele W. Magnitude of glycemic control and its associated factors among patients with type 2 diabetes at Tikur Anbessa Specialized Hospital, Addis Ababa, Ethiopia. PLoS One. 2018;13(3):e0193442.

32. Fiseha T, Alemayehu E, Kassahun W, Adamu A, Gebreweld A. Factors associated with glycemic control among diabetic adult out-patients in Northeast Ethiopia. BMC Res Notes. 2018;11(1): 316.
33. Abebe SM, Berhane Y, Worku A, Alemu S, Mesfin N. Level of sustained glycemic control and associated factors among patients with diabetes mellitus in Ethiopia: a hospital-based cross-sectional study. Diabetes Metab Syndr Obes Targets Ther. 2015;8:65.

34. Mideksa S, Ambachew S, Biadgo B, Baynes HW. Glycemic control and its associated factors among diabetes mellitus patients at Ayder Comprehensive Specialized Hospital, Mekelle-Ethiopia. Adipocyte. 2018;7(3):197-203.

35. Kassahun T, Eshetie T, Gesesew H. Factors associated with glycemic control among adult patients with type 2 diabetes mellitus: a cross-sectional survey in Ethiopia. BMC Res Notes. 2016;9(1):78.

36. Abejew AA, Belay AZ, Kerie MW. Diabetic complications among adult diabetic patients of a tertiary hospital in Northeast Ethiopia. Adv Public Health. 2015;2015:290920.

37. Khattab M, Khader YS, Al-Khawaldeh A, Ajlouni K. Factors associated with poor glycemic control among patients with type 2 diabetes. J Diabetes Complic. 2010;24(2):84-9.

38. Davies M. The reality of glycaemic control in insulin treated diabetes: defining the clinical challenges. Int J Obes. 2004;28(S2):S14.

39. American Diabetes Association. Standards of medical care in Diabetes-2018. Diabetes Care J Clin Appl Res Educ. 2018;41(Suppl 1):S13-28.

40. Toobert DJ, Hampson SE, Glasgow RE. The summary of diabetes self-care activities measure. Diabetes Care. 2000;23(7):943-50.

41. Garcia AA, Villagomez ET, Brown SA, Kouzekanani $\mathrm{K}$, Hanis CL. The Starr County Diabetes Education Study: development of the Spanish-language diabetes knowledge questionnaire. Diabetes Care. 2001;24(1):16-21.

42. Pantalone KM, Hobbs TM, Wells BJ, et al. Clinical characteristics, complications, comorbidities and treatment patterns among patients with type 2 diabetes mellitus in a large integrated health system. BMJ Open Diabetes Res Care. 2015;3(1): e000093.

43. Tegegn HG, Erku DA, Sebsibe G, et al. Medicationrelated quality of life among Ethiopian elderly patients with polypharmacy: a cross-sectional study in an Ethiopia university hospital. PLoS One. 2019;14(3):e0214191.

44. NHLBI Obesity Education Initiative. The practical guide: identification, evaluation, and treatment of overweight and obesity in adults. National Institutes of Health (NIH Publication 00-4084); 2000. 
https://www.nhlbi.nih.gov/files/docs/guidelines/ prctgd_c.pdf. Accessed 28 Jan 2019.

45. Alsulaiman TA, Al-Ajmi HA, Al-Qahtani SM, et al. Control of type 2 diabetes in King Abdulaziz Housing City (Iskan) population, Saudi Arabia. J Family Community Med. 2016;23(1):1.

46. Kayar Y, Ilhan A, Kayar NB, et al. Relationship between the poor glycemic control and risk factors, life style and complications. Biomed Res India. 2017;28(4):1581-6.

47. Kibirige D, Akabwai GP, Kampiire L, Kiggundu DS, Lumu W. Frequency and predictors of suboptimal glycemic control in an African diabetic population. Int J Gen Medi. 2017;10:33.

48. Mendes ABV, Fittipaldi JAS, Neves RCS, Chacra AR, Moreira ED. Prevalence and correlates of inadequate glycaemic control: results from a nationwide survey in 6,671 adults with diabetes in Brazil. Acta Diabetol. 2010;47(2):137-45.

49. Otieno C, Kariuki M. Quality of glycaemic control in ambulatory diabetics at the out-patient clinic of Kenyatta National Hospital, Nairobi. E Afr Med J. 2003;80(8):406-10.

50. De P, Banu S, Muthukumar D. Predictors of poor glycemic control in type 2 diabetic patients in
South Indian population. Int $\mathrm{J}$ Res Med Sci. 2018;6(2):545-50.

51. Rhee JJ, Ding VY, Rehkopf DH, Arce CM, Winkelmayer WC. Correlates of poor glycemic control among patients with diabetes initiating hemodialysis for end-stage renal disease. BMC Nephrol. 2015;16(1):204.

52. Tsuda A, Ishimura E, Inaba M. Response to comment on Tsuda et al. Poor glycemic control is a major factor in the overestimation of glomerular filtration rate in diabetic patients. Diabetes Care. 2014;37:596-603. https://doi.org/10.2337/dc132869.

53. Naderpoor N, Lyons JG, Mousa A, et al. Higher glomerular filtration rate is related to insulin resistance but not to obesity in a predominantly obese non-diabetic cohort. Sci Rep. 2017;7:45522.

54. Al-Hayek AA, Robert AA, Alzaid AA, et al. Association between diabetes self-care, medication adherence, anxiety, depression, and glycemic control in type 2 diabetes. Saudi Med J. 2012;33(6):681-3.

55. Demoz GT, Gebremariam A, Yifter H, $\mathrm{t}$ al. Predictors of poor glycemic control among patients with type 2 diabetes on follow-up care at a tertiary healthcare setting in Ethiopia. BMC Res Notes. 2019;12(1):207. 